\title{
Tiled Reconstruction Improves Structured Illumination Microscopy
}

David P. Hoffman*\& and Eric Betzig*

\section{Abstract:}

Structured illumination microscopy (SIM) is widely used for fast, long-term, live-cell superresolution imaging. However, SIM images can contain substantial artifacts if the sample does not conform to the underlying assumptions of the reconstruction algorithm. Here we describe a simple, easy to implement, process that can be combined with any reconstruction algorithm to alleviate many common SIM reconstruction artifacts and briefly discuss possible extensions.

\section{Main Text:}

Among super-resolution methods, structured illumination microscopy (SR-SIM) ${ }^{1}$ stands out for its ability to image live cells in multiple colors and at high speed with minimal phototoxicity over large fields of view (FOV). ${ }^{2-4}$ However, one criticism often leveled against SR-SIM ${ }^{5}$ is that its reconstruction algorithm employs both experimental and user-defined parameters which, if inaccurately measured or poorly chosen, can lead to image artifacts. ${ }^{6}$ For example, even measurement errors as small as $0.2 \%$ in SR-SIM illumination parameters (direction, phase, period, and modulation amplitude) have been shown to lead to substantial artifacts.,

Traditional SR-SIM ${ }^{7}$ assumes that the reconstruction parameters remain constant over the entire FOV. However, in practice optical aberrations can locally perturb illumination parameters and the detection point spread function (PSF) while differences in thickness or volumetric fluorescence density within the sample can lead to spatial variations in the optimal values of user-defined parameters for noise filtering, apodization, and discrete spatial frequency suppression needed to minimize artifacts. ${ }^{6}$ Spatial variation of the PSF is also commonly 
encountered in deconvolution ${ }^{8}$ where one stratagem to minimize artifacts has been to divide the FOV into subregions over which the PSF is assumed or known to be nearly constant. ${ }^{9,10}$ Here we apply tiled reconstruction, which has been used to reduce stripe artifacts in optical sectioning SIM ${ }^{11}$ to SR-SIM by dividing each raw SR-SIM data set into overlapping tiled subsets. Each subset can be reconstructed with independently measured or user-optimized parameters and then reassembled into a final, blended SR-SIM image covering the original FOV (bottom of Supplementary Fig. 1).

To illustrate, we collected cryogenic SR-SIM ${ }^{12}$ data of a high pressure frozen cell expressing the endoplasmic reticulum (ER) lumen marker mEmerald-ER3, and compared standard (Fig. 1a, top) and tiled (Fig. 1a, bottom) reconstruction. In many regions the reconstructed images were similar (Fig. 1b), but in others conventional reconstruction yielded artifacts not apparent with the tiled approach. Spatial maps of the illumination parameters across all tiles revealed particularly large variations in the standing wave modulation amplitude (Supplementary Fig. 2, right column) which, when approximated with a single global value in conventional reconstruction, could lead to such artifacts.

Tiled reconstruction introduces a new parameter, tile size. While smaller tile sizes ensure less variation in the reconstruction parameters across each tile, there is a practical limit below which the effects of pixilation become large and degrade the resulting images (right column of Supplementary Fig. 3). This occurs when, in Fourier space, the number of pixels in the overlap region between the diffraction limited information region and the frequency shifted information regions containing super-resolution spatial frequencies become too small to be stitched together accurately (Supplementary Fig. 4). Although we found that even 16-pixel tiles can produce accurate reconstructions (column 5 of Supplementary Fig. 3), in practice 64-pixel tiles offer a good compromise between minimizing artifacts from spatial variations in reconstruction parameters and maximizing reconstruction robustness and computational efficiency. 
We can envision a few extensions to tiled reconstruction. First, thicker specimens might benefit from tiling in 3D rather than only laterally as shown here. Second, square tile shapes are arbitrary, and fewer tiles might be needed if chosen on the basis of sample characteristics leading to spatially varying reconstruction parameters, such as sample thickness, proximity to the nucleus, or local fluorescence density. Third, if sample-induced aberrations affecting the detection pathway spatially vary, one could use a locally measured PSF for each tile during reconstruction. Regardless, we have found that tiled reconstruction is a simple and effective expedient for reducing artifacts in SR-SIM images that should benefit the broader SR-SIM community.
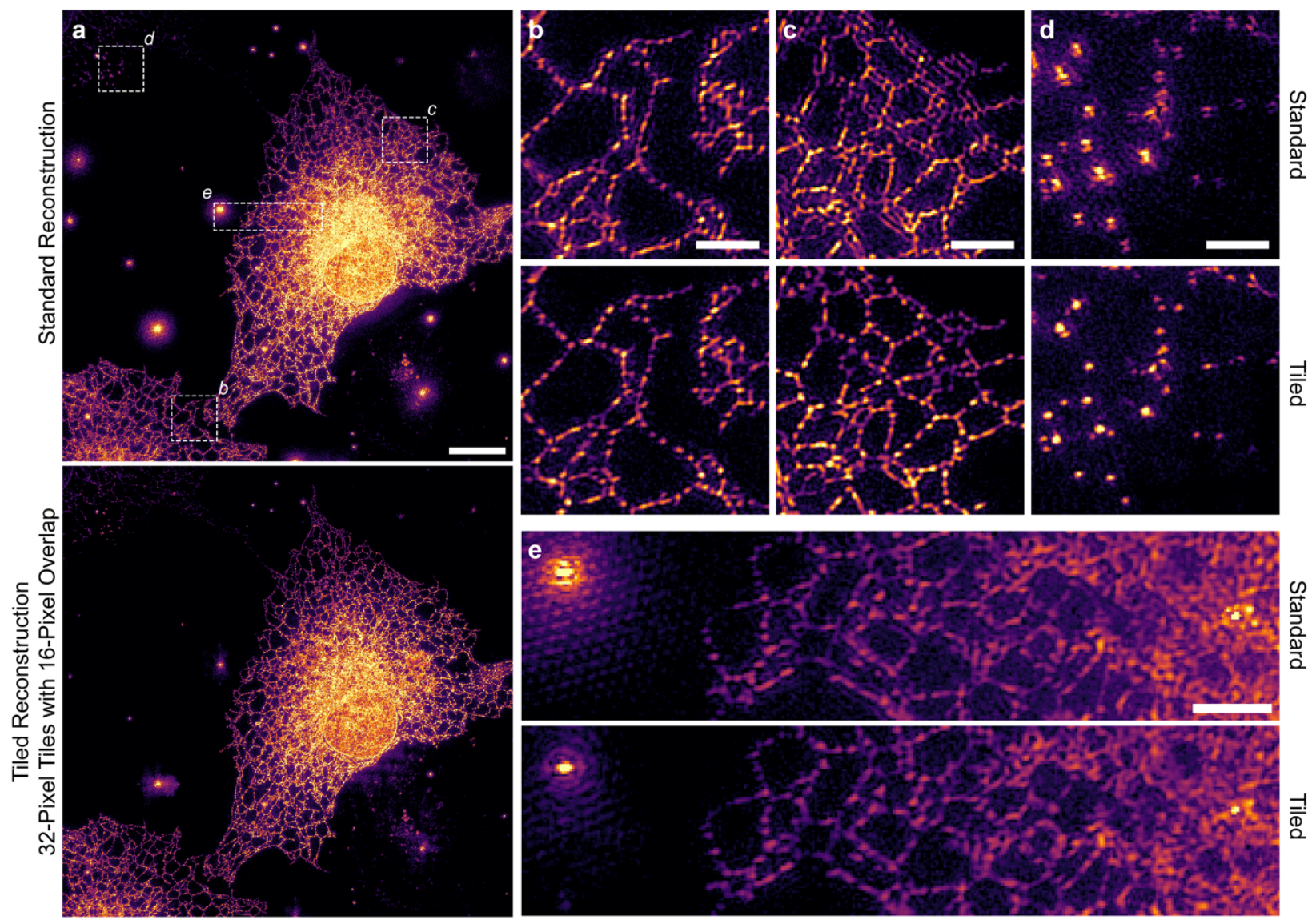

Fig. 1: Tiled SR-SIM reconstruction minimizes common SR-SIM artifacts. (a, top) Maximum intensity projection (MIP) through a standard 3D-SR-SIM reconstruction of a high pressure 
frozen COS-7 cell expressing an ER luminal marker. (a, bottom) Tiled reconstruction of the same cell using 1024 tiles, each 32 x 32-pixels with 16-pixel overlap. Scalebar, $10 \mu \mathrm{m}$. (b) An area of the cell with accurate reconstruction by both standard (top) and tiled (bottom) approaches. (c) An area of the cell exhibiting a "ghosting" artifact, under standard reconstruction (top) that is not present under tiled reconstruction (bottom). (d) Elimination of ringing artifacts at puncta through tiled reconstruction. (e) Crosshatch artifacts common in bright structures are effectively suppressed by tiled reconstruction (bottom). Colormaps are scaled identically for each pair of images. Scalebars, $2 \mu \mathrm{m}$.

\section{References}

1. Heintzmann, R. \& Huser, T. Super-Resolution Structured Illumination Microscopy. Chem. Rev. 117, 13890-13908 (2017).

2. Li, D. et al. Extended-resolution structured illumination imaging of endocytic and cytoskeletal dynamics. Science 349, aab3500 (2015).

3. Huang, X. et al. Fast, long-term, super-resolution imaging with Hessian structured illumination microscopy. Nature Biotechnology 36, 451-459 (2018).

4. Guo, Y. et al. Visualizing Intracellular Organelle and Cytoskeletal Interactions at Nanoscale Resolution on Millisecond Timescales. Cell 175, 1430-1442.e17 (2018).

5. Sahl, S. J. et al. Comment on "Extended-resolution structured illumination imaging of endocytic and cytoskeletal dynamics". Science 352, 527.1-527 (2016).

6. Demmerle, J. et al. Strategic and practical guidelines for successful structured illumination microscopy. Nature Protocols 12, 988-1010 (2017).

7. Gustafsson, M. G. L. et al. Three-Dimensional Resolution Doubling in Wide-Field Fluorescence Microscopy by Structured Illumination. Biophysical Journal 94, 4957-4970 (2008). 
8. Lucy, L. B. An iterative technique for the rectification of observed distributions. The Astronomical Journal 79, 745 (1974).

9. Trussell, H. \& Hunt, B. Sectioned methods for image restoration. IEEE Transactions on Acoustics, Speech, and Signal Processing 26, 157-164 (1978).

10. Temerinac-Ott, M., Ronneberger, O., Nitschke, R., Driever, W. \& Burkhardt, H. Spatiallyvariant Lucy-Richardson deconvolution for multiview fusion of microscopical 3D images. in 2011 IEEE International Symposium on Biomedical Imaging: From Nano to Macro 899-904 (2011). doi:10.1109/ISBI.2011.5872549.

11. Liu, Y., Lauderdale, J. D. \& Kner, P. Stripe artifact reduction for digital scanned structured illumination light sheet microscopy. Opt. Lett., OL 44, 2510-2513 (2019).

12. Hoffman, D. P. et al. Correlative three-dimensional super-resolution and block face electron microscopy of whole vitreously frozen cells. bioRxiv 773986 (2019) doi:10.1101/773986.

\section{Acknowledgements}

We thank M. Freeman for preparing the samples, G. Shtengel for help in constructing the microscope, and L. Shao and D. Li for SR-SIM assistance. mEmerald-ER3 were a gifts from Michael Davidson (Addgene plasmid \#54082; http://n2t.net/addgene:54082; RRID:Addgene_54082).

\section{Author information}

\section{Affiliations}

HHMI Janelia Research Campus, Ashburn, VA 20147, USA

David P. Hoffman \& Eric Betzig

Department of Molecular and Cell Biology, University of California, Berkeley, CA 94720, USA. 
Department of Physics, University of California, Berkeley, CA 94720, USA.

Howard Hughes Medical Institute, Berkeley, CA 94720, USA.

Helen Wills Neuroscience Institute, Berkeley, CA 94720, USA.

Molecular Biophysics and Integrated Bioimaging Division, Lawrence Berkeley National Laboratory, Berkeley, CA 94720, USA.

Eric Betzig

${ }^{\&}$ Current Affiliation: Eikon Therapeutics, Inc. Hayward, CA 94545, USA

\section{Contributions}

D.P.H. conceived the concept, collected and processed the data. E.B. supervised the project and wrote the correspondence together with D.P.H.

\section{Corresponding authors}

Correspondence to dave.p.hoffman@gmail.com, betzige@janelia.hhmi.org

\section{Ethics declarations}

\section{Competing interests}

The authors declare no competing interests. 